Supporting information

\title{
Surface Charge Controls the Sub-Organ Biodistributions of Gold Nanoparticles
}

Sukru Gokhan Elci, Ying Jiang, Bo Yan, Sung Tae Kim, Krishnendu Saha, Daniel F. Moyano, Gulen Yesilbag Tonga, Liam C. Jackson, Vincent M. Rotello and Richard W. Vachet*

Department of Chemistry, University of Massachusetts, 710 North Pleasant Street, Amherst, MA, 01002

* Address correspondence to rwvachet@chem.umass.edu 
Materials. All the reagents required for the AuNPs syntheses were purchased from Fisher Scientific or Sigma-Aldrich, with the exception of chloroauric acid, which was obtained from Strem Chemicals Inc. The 8-10 weeks old Balb/C type mice required for the animal experiments were obtained from Jackson Laboratory (Bar Harbor, ME). Animals were housed in the University of Massachusetts Amherst Animal Care facility. All of the animal experiments were conducted in accordance with the guidelines of the Institutional Animal Care and Use Committee (IACUC) at the university. Food and water intake of the mice were assessed. Chicken breast and beef liver were purchased from a local market (Big Y). The H\&E staining kit, nitric acid, hydrochloric acid and hydrogen peroxide were purchased from Fisher Scientific. The daily performance solution and Au standard were purchased from Perkin Elmer.

Gold Nanoparticle Synthesis and Characterization. Using the Brust-Schiffrin twophase synthesis method, $2 \mathrm{~nm}$ core AuNPs were synthesized. ${ }^{1}$ The AuNPs are initially synthesized with pentanethiols as the capping monolayer. Once these AuNPs were synthesized, the Murray place exchange method was used to functionalize the AuNPs with desired ligand functionality. ${ }^{2,3}$ Detailed syntheses of the ligands that were used in this work can be found in previous work. ${ }^{4}$ The place exchange reaction involved taking $10 \mathrm{mg}$ of the pentanethiolconjugated AuNPs and mixing with $30 \mathrm{mg}$ of the ligand of interest in a mixture of dry dichloromethane $(3 \mathrm{~mL})$ and methanol $(1 \mathrm{~mL})$. This mixture was stirred under nitrogen $\left(\mathrm{N}_{2}\right)$ for 3 days at $25{ }^{\circ} \mathrm{C}$. Then, the precipitate was collected (place-exchanged particles precipitate out) and dissolved in distilled water and dialyzed for three days to remove excess ligands, pentanethiol, and other salts present in the nanoparticle solution. The products of the synthesis, including the ligands and the final nanoparticles, were characterized using NMR and mass spectrometry. Figure 1 represents the structure of the AuNPs used in the study. For the characterization of AuNPs, the core sizes of the NPs were measured by transmission electron microscopy (TEM) on a JEOL100S electron microscope and were found to have core diameters of $2.1 \pm 0.2 \mathrm{~nm}$ (AuNP 1), $2.0 \pm 0.1 \mathrm{~nm}$ (AuNP 2), $1.8 \pm 0.2 \mathrm{~nm}$ (AuNP 3) and $2.0 \pm 0.4 \mathrm{~nm}$ (AuNP 4) (Figure S1). The AuNPs were also characterized by laser-desorption/ionization mass spectrometry (LDI-MS) to confirm the monolayer coating. ${ }^{5}$ Hydrodynamic size and the zeta potential of the particles were measured with Malvern NanoZetaSizer $(1 \mu \mathrm{M}$ NP concentration in $5 \mathrm{mM}$ phosphate buffer $\mathrm{pH}=7.4)$. 

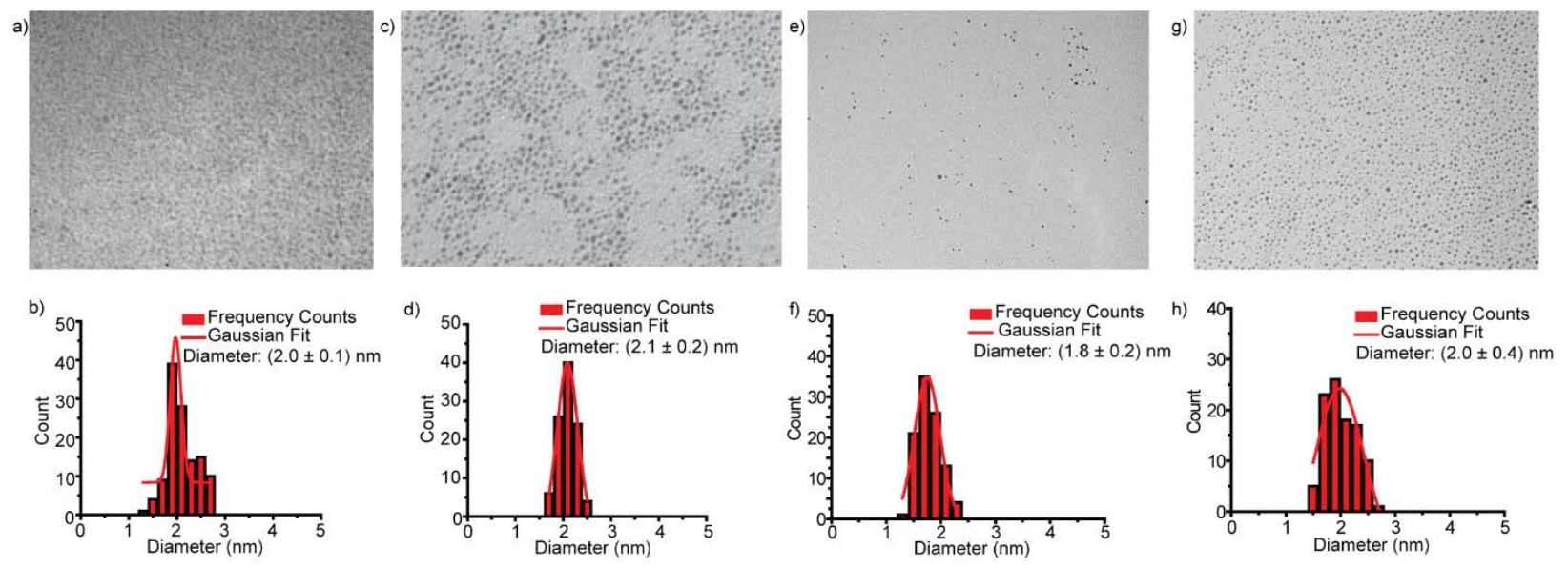

i) \begin{tabular}{|c|c|c|c|c|}
\hline & AuNP 1 & AuNP 2 & AuNP 3 & AuNP 4 \\
\hline TEM $(\mathrm{nm})$ & $2.0 \pm 0.1$ & $2.1 \pm 0.2$ & $1.8 \pm 0.2$ & $2.0 \pm 0.4$ \\
\hline Hydrodynamic size $(\mathrm{nm})$ & $10.0 \pm 0.7$ & $9.2 \pm 0.8$ & $13.5 \pm 4.3$ & $7.0 \pm 0.2$ \\
\hline ל-potential $(\mathrm{mV})$ & $21.1 \pm 0.5$ & $16.6 \pm 0.6$ & $-0.8 \pm 7.6$ & $-41.2 \pm 1.7$ \\
\hline
\end{tabular}

Figure S1. TEM images of the AuNPs studied in this work and summary of the TEM, DLS and zeta potential measurements of the particles.

Preparation of homogenates for matrix-matched standards: Purchased chicken breast tissue was cut into small pieces and placed in a 15-mL plastic centrifuge tubes to be homogenized. Water was added into each tube until it covered all the breast tissue, and the mixture was homogenized using a PowerGen 125 homogenizer (Fisher Scientific). Then, the homogenized tissues were transferred into $1.5 \mathrm{~mL}$ centrifuge tubes and centrifuged at $12000 \mathrm{rpm}$ for 10 mins. Water was removed after centrifugation and the homogenates were stored in the freezer until their use.

Preparation of sampler holder for matrix-matched standards: 50-mL plastic tubes were cut at the 35-mL mark, and the top part of the tube with its cap was used as the base. An optimal cutting temperature (OCT) solution was added to the tube. Five Edvotek $(0.5-10 \mu L)$ ultra pipet tips were attached to a piece of tape and slowly placed into the OCT solution. The setup then was placed in a freezer until it was completely frozen. Then, the embedded tips were removed using a tweezer and five different concentrations of the gold nanoparticle-tissue homogenate mixtures were transferred into the holes created by the tips. Figure S2 illustrates the total procedure of the matrix matched sample preparation. 
Matrix-matched standard preparation for LA-ICP-MS. Chicken breast and beef liver were purchased from a local market and used as the matrix-matched standards for spleen and liver, respectively. Details of the homogenization process can be found in the Supporting Information. After homogenization, $50 \mathrm{mg}$ of the homogenates were weighed and mixed with 50 $\mu \mathrm{L}$ of varied concentration of AuNPs. The preparation of the matrix matched samples is illustrated in Figure S2. The mixtures were then transferred into a homemade sample holder to be sliced into the desired thickness using the cryostat microtome. Details of the sample holder and concentration of the AuNPs used can be found in Supporting Information. This matrix match quantification strategy allowed us to quantify the amounts of the Au in these organs upon LAICP-MS imaging.

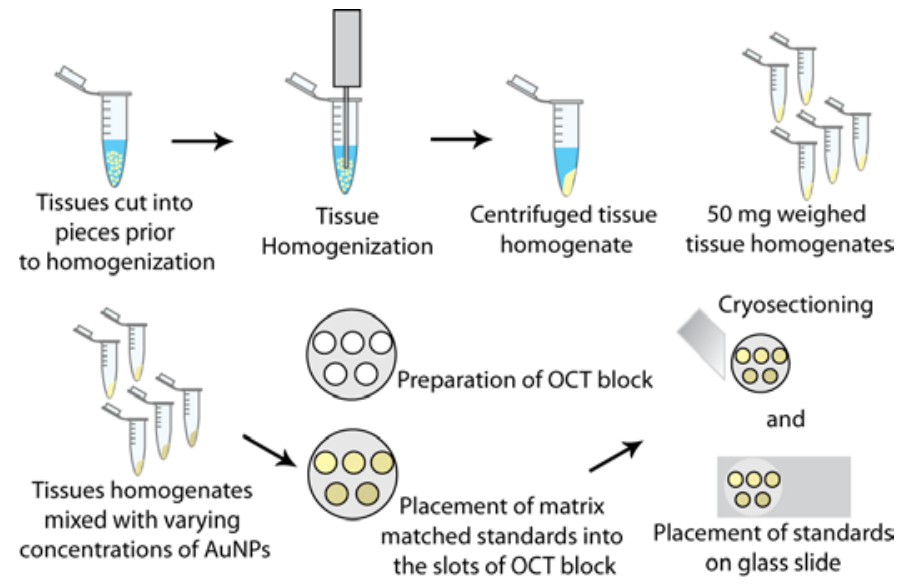

Figure S2. Illustration of the matrix matched standard preparation for LA-ICP-MS imaging

Gold percentage determination in spleen tissue regions. The gold concentration in each pixel of the red pulp was determined after comparison to a calibration curve as described in the experimental section. The concentrations from each pixel were then summed to obtain the total gold concentration in the red pulp region, and the percentage was calculated after summing the total gold from each of the three parts of the spleen. We determined the amount in each marginal zone by summing the gold concentrations in a single pixel area surrounding each white pulp region. The gold concentrations were obtained via comparison to a calibration curve, and the reported percentage was calculated in the same manner as with the red pulp. The gold concentrations were obtained via comparison to a calibration curve, and the reported percentage was calculated in the white pulp in the same manner as with the red pulp and marginal zone. 


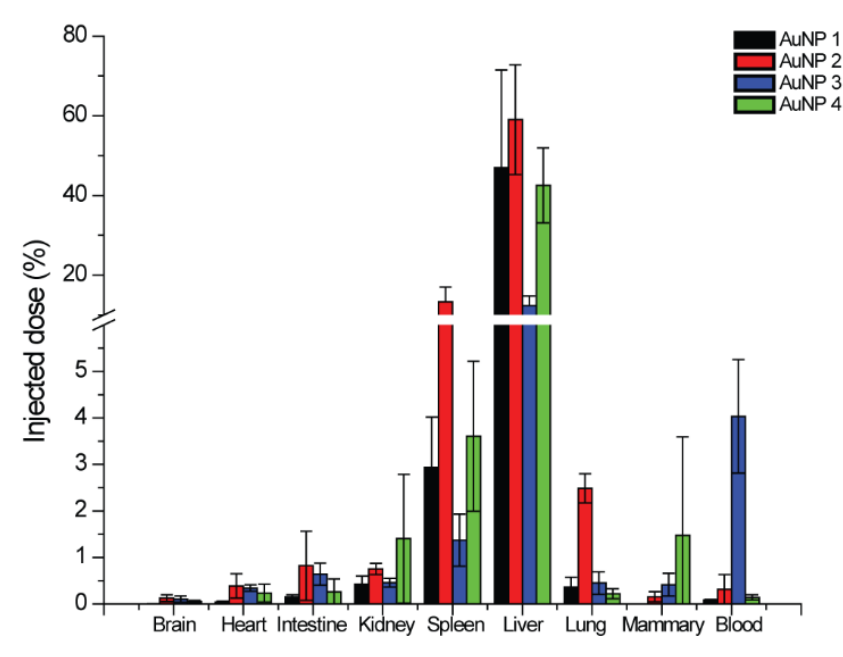

Figure S3. Injected AuNP doses in different organs after 24 hours of injection.
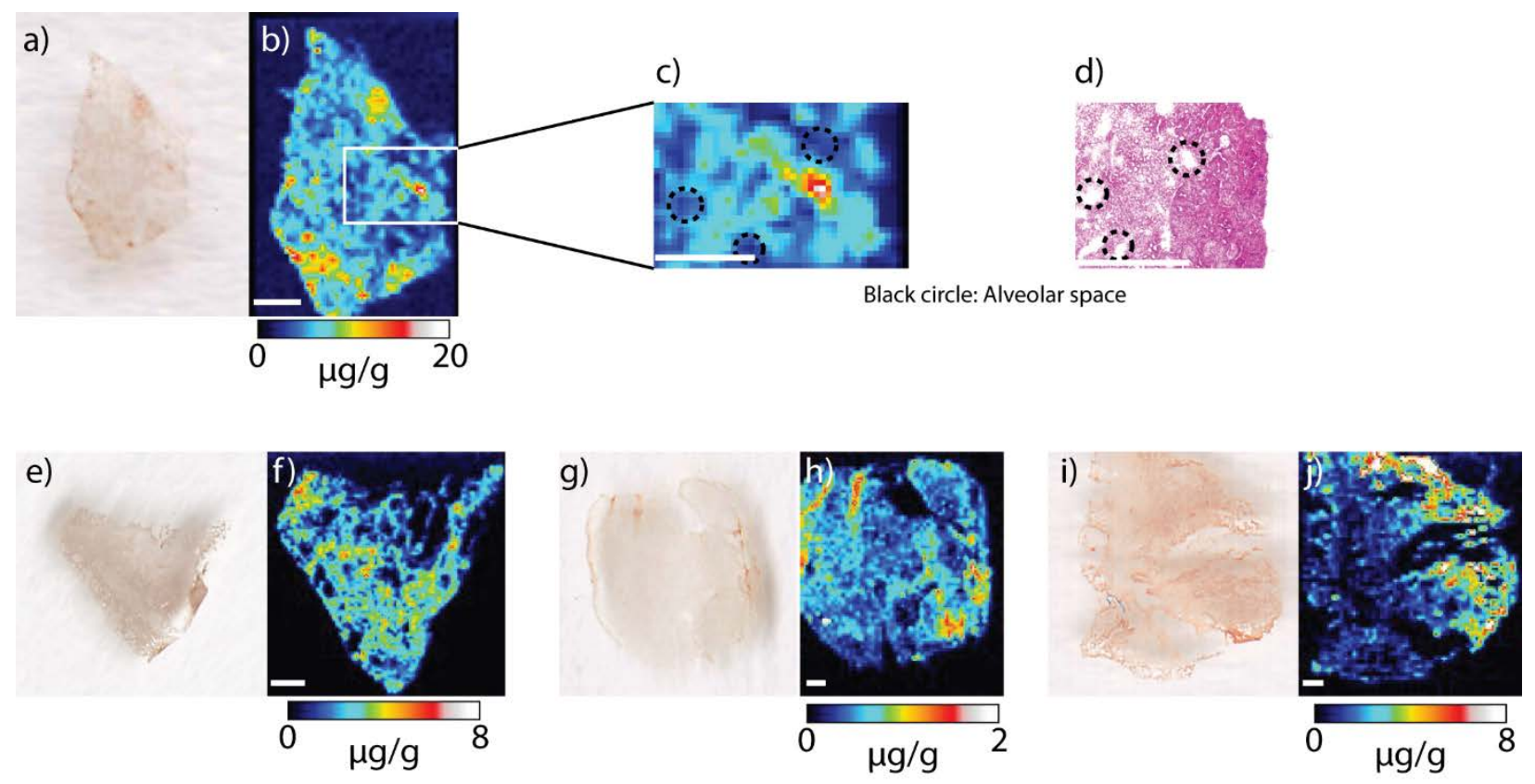

Figure S4. Imaging results for the lung tissues. (a) optical and (b) quantitative LA-ICP-MS images of AuNP 1; c) zoomed-in area illustrating the amount of AuNP 1 in a selected area of the lung tissue with various alveolar spaces indicated in black dotted lines; (d) optical image after H\&E staining of the same region shown in (c), indicating the alveolar spaces in black dotted lines. (e) optical and (f) quantitative LA-ICP-MS images of AuNP 2; (g) optical and (h) quantitative LA-ICP-MS images of AuNP 3 (i) optical and (j) quantitative LA-ICP-MS images of AuNP 4. All scale bars correspond to $0.5 \mathrm{~mm}$. 


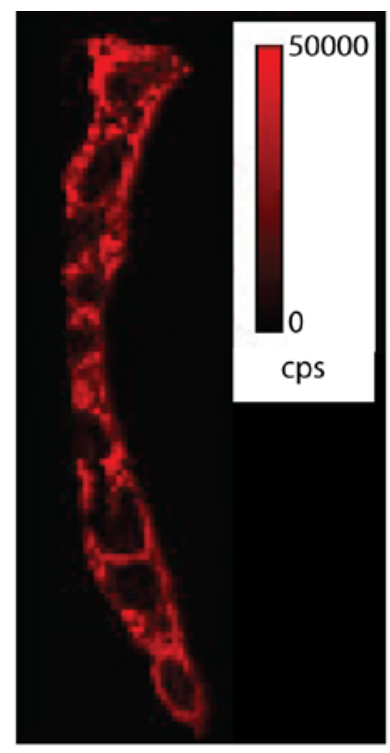

AuNP1

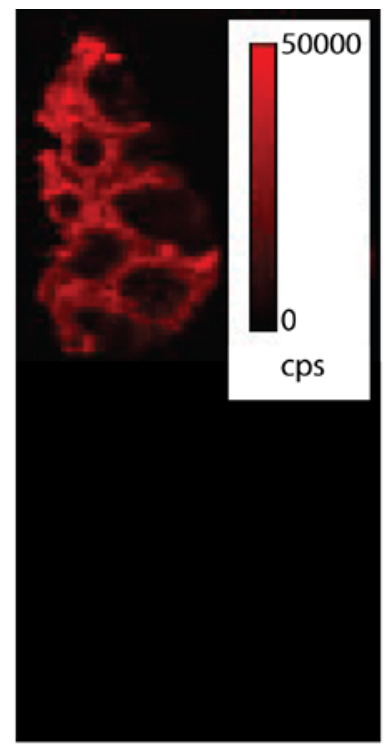

AuNP2

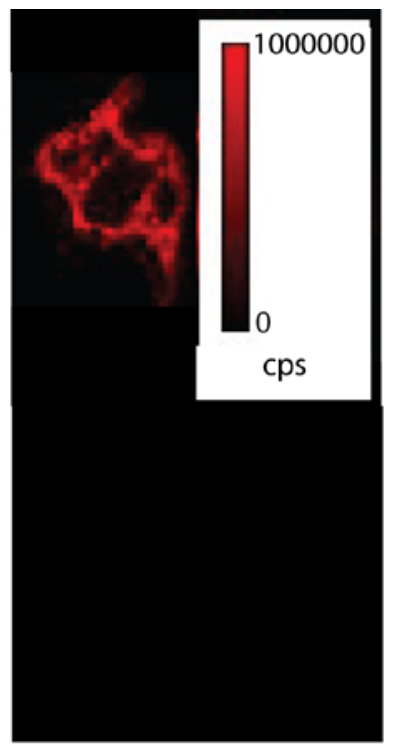

AuNP3

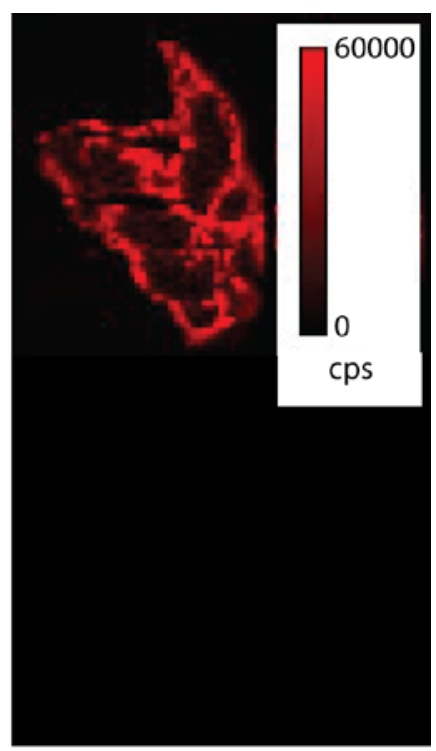

AuNP4

Figure S5. ${ }^{57} \mathrm{Fe}$ images of the spleen tissues. Higher concentrations of Fe are found in the red pulp as this region is infused with blood, whereas lower Fe concentrations are found in the white pulp.

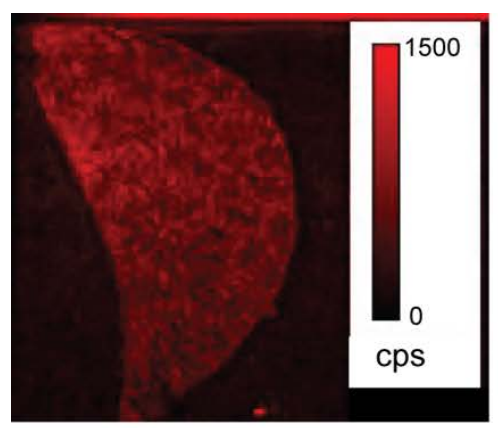

AuNP1

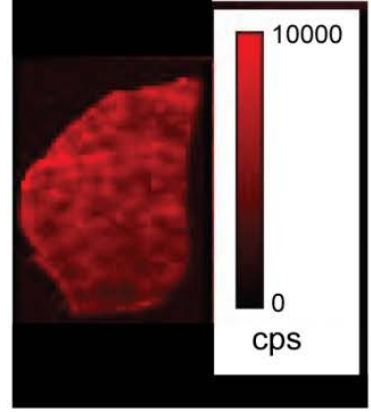

AuNP2

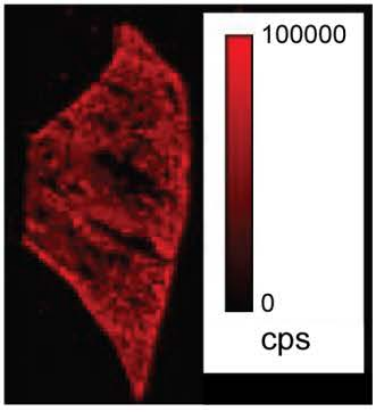

AuNP3

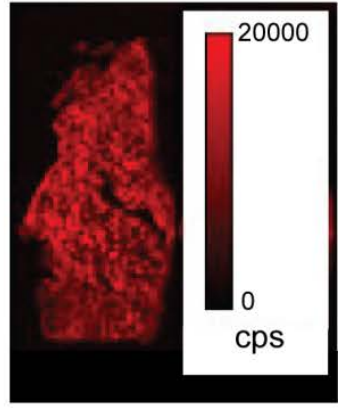

AuNP4

Figure S6. ${ }^{57} \mathrm{Fe}$ images of the liver tissues.

Table S1. ICP-MS instrument parameters

\begin{tabular}{ll}
\hline RF Power & $1.6 \mathrm{~kW}$ \\
\hline Nebulizer gas flow rate (Ar) & $0.7 \mathrm{~L} / \mathrm{min}$ \\
\hline Plasma gas flow rate (Ar) & $16.5 \mathrm{~L} / \mathrm{min}$ \\
\hline Auxiliary gas flow rate (Ar) & $1.4 \mathrm{~L} / \mathrm{min}$ \\
\hline Analog stage voltage & $-1600 \mathrm{~V}$ \\
\hline Pulse stage voltage & $950 \mathrm{~V}$ \\
\hline Deflector voltage & $-12 \mathrm{~V}$ \\
\hline Dwell time & $50 \mathrm{~ms}$ \\
\hline
\end{tabular}


Table S2. Laser ablation instrument parameters

\begin{tabular}{ll}
\hline Laser energy & $3.34 \mathrm{~J}$ \\
\hline Spot size & $50 \mu \mathrm{m}$ \\
\hline Scan rate & $10 \mu \mathrm{m} / \mathrm{s}$ \\
\hline Frequency & $10 \mathrm{~Hz}$ \\
\hline Carrier gas flow rate $(\mathrm{He})$ & $0.6 \mathrm{~L} / \mathrm{min}$ \\
\hline Shutter delay & $10 \mathrm{~s}$ \\
\hline
\end{tabular}

\section{References}

1 Brust, M.; Walker, M.; Bethell, D.; Schiffrin, D. J.; Whyman, R. Synthesis of Thiol-Derivatised Gold Nanoparticles in A Two-Phase Liquid-Liquid System. J. Chem. Soc., Chem. Commun. 1994, 801-802.

${ }^{2}$ Hong R.; Emrick T.; and Rotello V. M. Monolayer-Controlled Substrate Selectivity Using Noncovalent EnzymeNanoparticle Conjugates. J. Am. Chem. Soc. 2004, 126, 13572-13583.

${ }^{3}$ Jiang, Y.; Huo, S.; Mizuhara, T.; Das, R.; Lee, Y.-W.; Hou, S.; Moyano, D. F.; Duncan, B.; Liang, X.-J.; Rotello V. M. The Interplay of Size and Surface Functionality on the Cellular Uptake of Sub-10 nm Gold Nanoparticles. ACS Nano 2015, 9, 9986-9993.

${ }^{4}$ Miranda, O. R.; Chen, H.-T.; You, C.-C.; Mortenson, D. E.; Yang, X.-C.; Bunz, U. H. F.; Rotello, V. M. EnzymeAmplified Array Sensing of Proteins in Solution and in Biofluids J. Am. Chem. Soc. 2010, 132, 5285-5289.

${ }^{5}$ Yan, B.; Zhu, Z. J.; Miranda, O. R.; Chompoosor, A.; Rotello, V. M.; Vachet, R. W. Laser Desorption/Ionization Mass Spectrometry Analysis of Monolayer-Protected Gold Nanoparticles. Anal. Bioanal. Chem. 2010, 396, 10251035. 